\title{
A NONCOMMUTATIVE AG INEQUALITY
}

\author{
IVICA GUSIĆ
}

Abstract. We give a proof of AG inequality in noncommutative linearly ordered rings.

Mathematics subject classification (2000): 06F25, 26D05.

Key words and phrases: noncommutative linearly ordered ring, AG inequality, quasi-sum of squares.

\section{REFERENCES}

[1] E. ARTIN, Geometric algebra, Intersc. Publ., INC., New York 1966.

[2] P. S. BULlen, Handbook of Means and Their Inequalities, Kluwer Academic Publishers, Dordrecht/Boston/London, 2003

[3] L. FuCHS, Partially ordered algebraic systems, Pergamon Press 1963.

[4] I. GusIĆ, A purely algebraic proof of AG inequality, Math. Ineq. Appl., 8, (2) (2005), 191-198.

[5] D. HILBERT, Grundlagen der Geometrie, Springer, 1913.

[6] A. HuRwITZ, Über den Vergleich des arithmetischen und des geometrischen Mittels, J. Reine Angew. Math., 108, (1891), 266-268.

[7] T. V. TARARYKOVA, An explicit representation a quasi-sum of squares of a polynomial generated by the AG inequality, Math. Ineq. Appl., 9, (4) (2006), 649-659. 